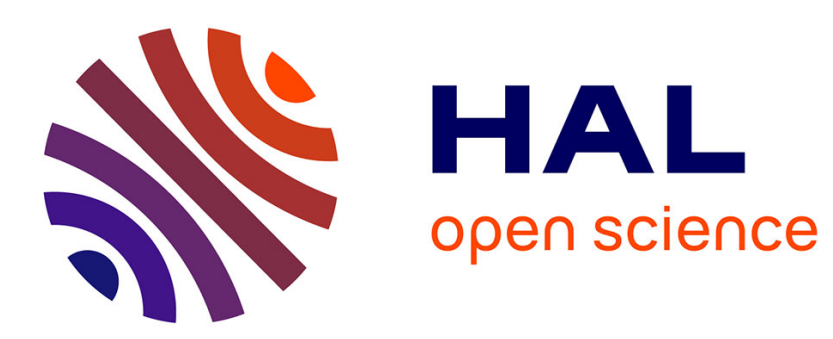

\title{
Mathematical Morphology on Hypergraphs: Preliminary Definitions and Results
}

\author{
Isabelle Bloch, Alain Bretto
}

\section{To cite this version:}

Isabelle Bloch, Alain Bretto. Mathematical Morphology on Hypergraphs: Preliminary Definitions and Results. Discrete Geometry for Computer Imagery 16th IAPR International Conference, DGCI 2011, Apr 2011, Nancy, France. pp.429-440. hal-01021442

\section{HAL Id: hal-01021442 https://hal.science/hal-01021442}

Submitted on 9 Jul 2014

HAL is a multi-disciplinary open access archive for the deposit and dissemination of scientific research documents, whether they are published or not. The documents may come from teaching and research institutions in France or abroad, or from public or private research centers.
L'archive ouverte pluridisciplinaire HAL, est destinée au dépôt et à la diffusion de documents scientifiques de niveau recherche, publiés ou non, émanant des établissements d'enseignement et de recherche français ou étrangers, des laboratoires publics ou privés. 


\title{
Mathematical Morphology on Hypergraphs: Preliminary Definitions and Results
}

\author{
Isabelle Bloch ${ }^{1}$ and Alain Bretto ${ }^{2, \star}$ \\ 1 Télécom ParisTech, CNRS LTCI, Paris, France \\ isabelle.bloch@telecom-paristech.fr \\ 2 Greyc Cnrs-Umr 6072, Bd Marechal Juin BP 5186, Caen, France \\ alain.bretto@info.unicaen.fr
}

\begin{abstract}
In this article we introduce mathematical morphology on hypergraphs. We first define lattice structures and then mathematical morphology operators on hypergraphs. We show some relations between these operators and the hypergraph structure, considering in particular duality and similarity aspects.
\end{abstract}

\section{Introduction}

Mathematical morphology is a widely used theory for contemporary information processing in various lattice frameworks. Concerning structural information processing, mathematical morphology has been developed on graphs [6]10]15]16], but nothing has been done yet on hypergraphs to the best of our knowledge.

Hypergraphs were introduced in the $60 \mathrm{~s}$ as a generalization of graphs 1], where edges become hyperedges and can connect more than two vertices, and have then been intensively studied. They have shown their interest in various fields such as computer science, game theory, databases, data mining, optimization [17, image processing and segmentation [45].

The aim of this paper is to propose preliminary definitions and results in this context. We consider an hypergraph defining the underlying space: $\mathcal{H}=(\mathcal{V}, \mathcal{E})$ with $\mathcal{V}$ the set of vertices and $\mathcal{E}$ the set of hyperedges. The powerset of $\mathcal{V}$ and $\mathcal{E}$ are denoted by $\mathcal{P}(\mathcal{V})$ and $\mathcal{P}(\mathcal{E})$, respectively. We denote a hypergraph by $H=(V, E)$ with $V \subseteq \mathcal{V}$ and $E \subseteq \mathcal{E}$.

After introducing some more notations and basic concepts related to hypergraphs in Section 2, our first objective (Section 3) is to define a lattice structure $(\mathcal{T}, \preceq)$ on the hypergraphs of $\mathcal{H}$, with $\preceq$ a partial ordering on $\mathcal{T}$ such that $(\mathcal{T}, \preceq$ ) is a complete lattice (suitable structure for mathematical morphology, as shown in 3778 11]). Mathematical morphology operators on hypergraphs are then defined in Section 4. Then, in Section 5, we consider dual hypergraphs and establish some links between morphological dilations and hypergraph duality concepts. Finally, in Section 6 we propose a simple example for computing similarity between hypergraphs based on dilations.

\footnotetext{
* This work was partially funded by a grant from Institut Télécom / Télécom Paris-

Tech, and was done during the sabbatical stay of A. Bretto at Télécom ParisTech.
} 


\section{Basic Concepts on Hypergraphs [1]}

A hypergraph $H$ denoted by $H=\left(V, E=\left(e_{i}\right)_{i \in I}\right)$ on a finite set $V$ is a family (which can be a multi-set) $\left(e_{i}\right)_{i \in I}$, (where $I$ is a finite set of indices) of subsets of $V$ called hyperedges. Sometimes we will denote $V$ as $V(H)$, and $E$ as $E(H)$. Let $\left(e_{j}\right)_{j \in\{1,2, \ldots l\}}$ be a sub-family of hyperedges of $E$. The set of vertices belonging to these hyperedges is denoted by $V\left(\cup_{j \in\{1,2, \ldots l\}} e_{j}\right)$, and $V(e)$ denotes the set of vertices forming the hyperedge $e$. When no confusion may arise, we will just denote by $e$ the set of vertices it contains. If $\bigcup_{i \in I} e_{i}=V$, the hypergraph is without isolated vertex (a vertex $x$ is isolated if $x \in V \backslash \bigcup_{i \in I} e_{i}$ ). By definition the empty hypergraph is the hypergraph $H_{\emptyset}$ such that $V=\emptyset$ and $E=\emptyset$.

Let $H=\left(V,\left(e_{i}\right)_{i \in I}\right)$ be a hypergraph. A induced subhypergraph $H\left(V^{\prime}\right)$ of $H$ with $V^{\prime} \subseteq V$ is a hypergraph defined as $H\left(V^{\prime}\right)=\left(V^{\prime},\left(e_{i} \cap V^{\prime}\right)_{e_{i} \cap V^{\prime} \neq \varnothing}\right)$. The partial hypergraph $H^{\prime}$ of $H$ generated by $J \subseteq I$ is the hypergraph $\left(V,\left(e_{j}\right)_{j \in J}\right)$. Given a subset $V^{\prime} \subseteq V$, a subhypergraph $H^{\prime}$ is the partial hypergraph $H^{\prime}=$ $\left(V^{\prime},\left\{e_{i}, i \in I \mid e_{i} \subseteq V^{\prime}\right\}\right)$. Without loss of generality we can suppose that the empty hypergraph, $H_{\emptyset}=(\emptyset, \emptyset)$ is a partial hypergraph, (resp. (induced) subhypergraph) of any hypergraph.

The star centered at $x$ is the set of hyperedges containing $x$, denoted by $H(x)$. The value $d(x)=|H(x)|$ is the degree of $x$.

If the family of hyperedges is a set of subsets of $V$, we say that $H$ is without repeated hyperedge i.e. $i \neq j \Longleftrightarrow e_{i} \neq e_{j}$. The rank of $H$ is the maximum cardinality of a hyperedge. A hypergraph is linear if $\left|e_{i} \cap e_{j}\right| \leq 1$ for $i \neq j$. A loop is a hyperedge with a cardinality equal to one. A simple hypergraph is a hypergraph $H=\left(V, E=\left(e_{i}\right)_{i \in I}\right)$ such that: $e_{i} \subseteq e_{j} \Longrightarrow i=j$.

The dual $H^{*}$ of a hypergraph without empty hyperedge and isolated vertex $H$ is a hypergraph whose set of vertices is isomorphic (denoted $\simeq$ ) to the set of hyperedges of $H$, and whose hyperedges are given by $X_{1}, X_{2}, \ldots X_{n}$ where $X_{j}=\left\{e_{i} \mid x_{j} \in e_{i}\right\}$. The transpose $A^{t}$ of the incidence matrix $A=\left(\left(a_{i j}\right)\right)$ of a hypergraph $H$ (i.e. $a_{i j}=1$ iff vertex $i$ belongs to hyperedge $j$ ) is the incidence matrix of $H^{*}=\left(V^{*} \simeq E, E^{*} \simeq(H(x))_{x \in V}\right)$ : for $v_{j}^{*} \in V^{*}$ and $e_{i}^{*} \in E^{*}, v_{j}^{*} \in e_{i}^{*}$ if and only if $a_{i j}=1$. Consequently $\left(H^{*}\right)^{*}=H$. Note that a hypergraph can be equivalently defined as a family (potentially multi-set) of hyperedges on a set of vertices, or as an incidence matrix.

A hypergraph $H=(V, E)$ is isomorphic to a hypergraph $H^{\prime}=\left(V^{\prime}, E^{\prime}\right)(H \simeq$ $\left.H^{\prime}\right)$, if there exist a bijection $f: V \rightarrow V^{\prime}$ and a permutation $\pi$ of $I$ such that: $f\left(V\left(e_{i}\right)\right)=a_{\pi(i)}$, for $e_{i} \in E$ and $a_{\pi(i)} \in E^{\prime}$. The mapping $f$ is then called isomorphism of hypergraphs. Note that $H \simeq H^{\prime}$ if and only if $H^{*} \simeq H^{\prime *}$.

Let $H=(V, E)$ be a hypergraph, $E=\left(e_{1}, e_{2}, \ldots, e_{m}\right)$. A path $P$ in $H$ from $x_{i_{1}}$ to $x_{i_{s+1}}$ is an alternated vertex-edge sequence $x_{i_{1}}, e_{i_{1}}, x_{i_{2}}, e_{i_{2}}, \ldots, x_{i_{s}}, e_{i_{s}}, x_{i_{s+1}}$ such that $\left\{x_{i_{k}}, x_{i_{k+1}}\right\} \in e_{i_{k}},\left(k=i_{1}, i_{2}, \ldots, i_{s}\right)$ and $x_{i_{k}} \neq x_{i_{j}}, e_{i_{k}} \neq e_{i_{j}}\left(i_{k} \neq i_{j}\right)$, where $s$ is called the length of path $P$. The distance between vertices $x$ and $y$, $d(x, y)$ is the minimum length among those of all paths which connect $x$ and $y$. If for each pair of vertices $(x, y)$ there is a path from $x$ to $y$, the hypergraph $H$ is said connected. 


\section{Lattice Structures on Hypergraphs}

In this section we define a few lattices on hypergraphs, as the basic algebraic structures on which mathematical morphology operators are then defined.

A lattice on the set of vertices can be simply defined by $\mathcal{T}=(\mathcal{P}(\mathcal{V}), \subseteq)$. This is the classical lattice defined on the powerset of a set, with the standard set inclusion as partial ordering. It is a complete lattice. However is it not really interesting since it does not say anything on the structure of the hypergraph.

A more interesting lattice can be defined, based on closed sets of vertices, involving the stars of vertices. Let $H=(V, E)$ be a hypergraph and let $V^{\prime} \subseteq V$. We say that $V^{\prime}$ is a closed set if $\forall(x, y) \in V^{\prime 2}, V(H(x) \cap H(y)) \subseteq V^{\prime}$. We denote by $\mathcal{C}(H)$ the family of closed sets with the empty set.

Proposition 1. The structure $(\mathcal{C}(H), \subseteq)$ is a complete lattice. The infimum is $\wedge=\cap$ and the supremum is: $\forall\left(V^{\prime}, V^{\prime \prime}\right) \in \mathcal{C}(H)^{2}, V^{\prime} \vee V^{\prime \prime}=\cap\left\{V^{\prime \prime \prime} \in \mathcal{C}(H) \mid V^{\prime} \cup\right.$ $\left.V^{\prime \prime} \subseteq V^{\prime \prime \prime}\right\}$, i.e. the intersection of all closed sets containing $V^{\prime} \cup V^{\prime \prime}$, and its extension to any family. The smallest element is $\emptyset$ and the largest element is $V$. Note that $\mathcal{C}(H)$ is a Moore family [3].

A lattice on the set of hyperedges can be defined by $\mathcal{T}=(\mathcal{P}(\mathcal{E}), \subseteq)$. Again it is a classical complete lattice on the powerset of a set and classical results can be used directly. The next definitions, on the hypergraphs themselves, are probably more interesting.

Lattices on the hypergraphs will allow us to better account for the whole structural information encoded in hypergraphs, considering both vertices and hyperedges in the definition of the lattice structure.

The simplest idea is to consider the inclusion on the powerset of vertices and edges, respectively. Other ideas could be to define a partial ordering based on the notions of induced sub-hypergraph, partial hypergraph and sub-hypergraph.

In all cases, $\mathcal{T}$ is defined as:

$$
H=(V, E) \in \mathcal{T} \Leftrightarrow\left\{\begin{array}{l}
V \subseteq \mathcal{V} \\
E \subseteq \mathcal{E} \\
\{x \in \mathcal{V} \mid \exists e \in E, x \in e\} \subseteq V
\end{array}\right.
$$

The last condition ensures that $H$ is actually a hypergraph, where the hyperedges are sets of vertices of $V$, and can be equivalently written as $\forall e \in E, V(e) \subseteq V$. There is no equivalent restriction on $V$ if isolated vertices are accepted.

Partial ordering based on the inclusion on the powersets of vertices and hyperedges

\section{Definition 1}

$$
\forall\left(H_{1}, H_{2}\right) \in \mathcal{T}^{2}, H_{1}=\left(V_{1}, E_{1}\right), H_{2}=\left(V_{2}, E_{2}\right), H_{1} \preceq H_{2} \Leftrightarrow\left\{\begin{array}{l}
V_{1} \subseteq V_{2} \\
E_{2} \subseteq E_{2}
\end{array}\right.
$$

This definition is similar to the one used in [6] for graphs. 
Proposition 2. The following properties hold:

- $\preceq$ defines a partial ordering on $\mathcal{T}$.

- The infimum is: $H_{1} \wedge H_{2}=\left(V_{1} \cap V_{2}, E_{1} \cap E_{2}\right)$, and for any family $\left(H_{i}\right)$ : $\bigwedge_{i} H_{i}=\left(\bigcap_{i} V_{i}, \bigcap_{i} E_{i}\right)$.

- The supremum is: $H_{1} \vee H_{2}=\left(V_{1} \cup V_{2}, E_{1} \cup E_{2}\right)$, and for any family $\left(H_{i}\right)$ : $\bigvee_{i} H_{i}=\left(\bigcup_{i} V_{i}, \bigcup_{i} E_{i}\right)$.

- $(\mathcal{T}, \preceq)$ is a complete lattice, which is moreover sup-generated. Its smallest element is $H_{\emptyset}=(\emptyset, \emptyset)$ and its largest element is $\mathcal{H}=(\mathcal{V}, \mathcal{E})$. We have $\bigvee \emptyset=\bigwedge \mathcal{T}=H_{\emptyset}$ and $\bigwedge \emptyset=\bigvee \mathcal{T}=\mathcal{H}$.

Note that it is not complemented (in order to have $E \cup E^{c}=\mathcal{E}$, we would have to consider in $E^{c}$ all hyperedges that are not in $E$, including those which have vertices both in $V$ and in $V^{c}$, so $\left(V^{c}, E^{c}\right)$ would not be a hypergraph in $\mathcal{T}$ ).

Partial ordering based on the notion of induced sub-hypergraph

Definition 2. A partial ordering can be defined from the type of inclusion which is implicit in the definition of induced sub-hypergraph, as:

$$
\forall\left(H_{1}, H_{2}\right) \in \mathcal{T}^{2}, H_{1} \preceq_{i} H_{2} \Leftrightarrow\left\{\begin{array}{l}
V_{1} \subseteq V_{2} \\
E_{1}=\left\{e \cap V_{1} \mid e \in E_{2}\right\}
\end{array}\right.
$$

i.e. $H_{1}$ is the sub-hypergraph induced by $H_{2}$ for $V_{1}$.

Proposition 3. The relation $\preceq_{i}$ is a partial ordering on $\mathcal{T}$.

It might be more suitable (to allow for more frequent comparisons between hypergraphs) to propose a less strict version where $E_{1}$ is only required to be included in the set of hyperdeges of the induced sub-hypergraph:

\section{Definition 3}

$$
\forall\left(H_{1}, H_{2}\right) \in \mathcal{T}^{2}, H_{1} \preceq_{i}^{\prime} H_{2} \Leftrightarrow\left\{\begin{array}{l}
V_{1} \subseteq V_{2} \\
E_{1} \subseteq\left\{e \cap V_{1} \mid e \in E_{2}\right\}
\end{array}\right.
$$

Proposition 4. The following properties hold:

- $\preceq_{i}^{\prime}$ is a partial ordering on $\mathcal{T}$.

$-\left(\mathcal{T}, \preceq_{i}^{\prime}\right)$ is a complete lattice.

- The infimum is: $\left.H_{1} \wedge_{i}^{\prime} H_{2}=\left(V_{1} \cap V_{2},\left\{e_{1} \cap V_{2}, e_{2} \cap V_{1} \mid e_{1} \in E_{1}, e_{2} \in E_{2}\right\} \cap \mathcal{E}\right\}\right)$, and a straightforward extension to any family $\left(H_{i}\right)$.

- The supremum is: $H_{1} \vee_{i}^{\prime} H_{2}=\left(V_{1} \cup V_{2}, E_{1} \cup E_{2}\right)$, and its extension to any family $\left(H_{i}\right)$.

- The smallest element is $H_{\emptyset}=(\emptyset, \emptyset)$ and the largest element is $\mathcal{H}=(\mathcal{V}, \mathcal{E})$.

Another idea involves isomorphisms, as in the following definition.

Definition 4. Let $\boldsymbol{H}$ be the set of isomorphism classes of hypergraphs. A partial order on $\boldsymbol{H}$ can be defined, for all $\mathrm{H}_{1}, \mathrm{H}_{2}$ in $\boldsymbol{H}$ as:

$H_{1} \leq_{f} H_{2} \Longleftrightarrow H_{1}$ is isomorphic (by $f$ ) to an induced subhypergraph of $H_{2}$ 
Proposition 5. The structure $\left(\boldsymbol{H}, \leq_{f}\right)$ is a complete lattice. The supremum is $\sup \left\{H_{1}, H_{2}\right\}=H_{1} \vee H_{2}$ (as in Proposition Q for $\preceq$ ), and the infimum inf $\left\{H_{1}, H_{2}\right\}$ is the maximum common induced subhypergraph (and their extension to any family).

Partial ordering based on the notion of partial hypergraph

\section{Definition 5}

$$
\forall\left(H_{1}, H_{2}\right) \in \mathcal{T}^{2}, H_{1} \preceq_{p} H_{2} \Leftrightarrow\left\{\begin{array}{l}
V_{1}=V_{2} \\
E_{1} \subseteq E_{2}
\end{array}\right.
$$

Proposition 6 . $\preceq_{p}$ is a partial ordering on $\mathcal{T}$, and $\left(\mathcal{T}, \preceq_{p}\right)$ is a complete lattice. This is simply a restriction of $\preceq$ by considering only the hypergraphs with the same set of vertices, so it will not be further considered.

Partial ordering based on the notion of sub-hypergraph

\section{Definition 6}

$$
\forall\left(H_{1}, H_{2}\right) \in \mathcal{T}^{2}, H_{1} \preceq_{s} H_{2} \Leftrightarrow\left\{\begin{array}{l}
V_{1} \subseteq V_{2} \\
E_{1}=\left\{e \mid e \in E_{2} \text { and } V(e) \subseteq V_{1}\right\}
\end{array}\right.
$$

Note that the condition on the hyperegdes is stronger than $E_{1} \subseteq E_{2}$ and we may have more hyperegdes.

Another possibility would be to define $\preceq_{s}^{\prime}$ by replacing the equality in the condition on the hyperedges by an inclusion (as for $\preceq_{i}^{\prime}$ ).

Proposition 7 . $\preceq_{s}$ and $\preceq_{s}^{\prime}$ are partial ordering on $\mathcal{T}$.

These partial orderings may be interesting when the notions of (induced) subhypergraphs are explicitly involved in the application at hand.

In the following, we use $\preceq$ for defining in a general way a partial ordering between two hypergraphs.

\section{Mathematical Morphology on Hypergraphs}

Algebraic Dilation and Erosion. Once we have a complete lattice, the whole algebraic apparatus of mathematical morphology applies.

Let $(\mathcal{T}, \preceq)$ and $\left(\mathcal{T}^{\prime}, \preceq^{\prime}\right)$ be two complete lattices (which can be any of those defined in Section 3, and do not need to be equal). All the following definitions and results are common to the general algebraic framework of mathematical morphology in complete lattices $3,7,8,11,13]$.

Definition 7. An operator $\delta: \mathcal{T} \rightarrow \mathcal{T}^{\prime}$ is a dilation if: $\forall\left(x_{i}\right) \in \mathcal{T}, \delta\left(\vee_{i} x_{i}\right)=$ $\vee_{i}^{\prime} \delta\left(x_{i}\right)$, where $\vee$ denotes the supremum associated with $\preceq$ and $\vee^{\prime}$ the one associated with $\preceq^{\prime}$. An operator $\varepsilon: \mathcal{T}^{\prime} \rightarrow \mathcal{T}$ is an erosion if: $\forall\left(x_{i}\right) \in \mathcal{T}^{\prime}, \varepsilon\left(\wedge_{i}^{\prime} x_{i}\right)=$ $\wedge_{i} \varepsilon\left(x_{i}\right)$, where $\wedge$ and $\wedge^{\prime}$ denote the infimum associated with $\preceq$ and $\preceq^{\prime}$, respectively.

All classical properties of mathematical morphology then hold 378], and are therefore not recalled here. 
Structuring Element and Morphological Operations. In classical morphology dilations and erosions can be expressed by means of a set, called structuring element, which defines a neighborhood at each point [12], and this idea has been used for graphs as well [16]. The structuring element "centered" at $x$ is $B_{x}=\delta(\{x\})$. More generally, the structuring element can be interpreted as a binary relation between two elements, thus enabling the extension of this idea to any lattice.

Defining morphological dilations on hypergraphs calls for canonical decompositions of the elements of the considered lattice.

In the case of the lattice $(\mathcal{P}(\mathcal{V}), \subseteq)$, each subset of vertices $V$ can be trivially decomposed as $V=\cup_{x \in V}\{x\}$, and a morphological dilation then writes $\delta_{B}(V)=$ $\cup_{x \in V} B_{x}=\cup_{x \in V} \delta(\{x\})$.

In the case of the lattice $(\mathcal{P}(\mathcal{E}), \subseteq)$, each subset of hyperedges $E$ can be decomposed as $E=\cup_{e \in E}\{e\}$, and a morphological dilation is then $\delta_{B}(E)=\cup_{e \in E} B_{e}=$ $\cup_{e \in E} \delta(\{e\})$.

Let us now consider the lattice of hypergraphs, with the partial ordering $\preceq$ (see Definition 1). Let $H=(V, E)$ be a hypergraph of this lattice. For $E$, a natural decomposition consists of $E=\vee_{e \in E}\{e\}$. For $V$ the decomposition should be consistent with the one of $E$, in order to associate an "elementary" hypergraph to each $e$. We thus consider $V(e)$, the set of vertices associated with $e$. Additionally, the decomposition should also involve all vertices that do not belong to any hyperedge. We denote by $V_{E}$ this set of vertices. Finally we propose the following canonical decomposition of $H$, from its sup generating property: $H=\left(\vee_{e \in E}(V(e),\{e\})\right) \vee\left(\vee_{x \in V_{E}}(\{x\}, \emptyset)\right)$.

The question of how the structuring element should be defined depends on the application and on the type of desired results. Examples are provided next.

Example 1. Let us consider $\mathcal{T}=(\mathcal{P}(\mathcal{E}), \subseteq)$. An example of structuring element, defining the elementary dilation of each hyperedge, consists in taking all hyperedges which have at least one vertex in common with the considered hyperedge:

$$
\forall e \in E, B_{e}=\delta(\{e\})=\left\{e^{\prime} \in \mathcal{E} \mid V(e) \cap V\left(e^{\prime}\right) \neq \emptyset\right\},
$$

where the intersection applies on the sets of vertices defining $e$ and $e^{\prime}$. Dilating a subset $E$ by this structuring element means adding all hyperedges that are directly connected to $E$.

As an illustration, let us consider the two hypergraphs depicted in Figure 1 . For the first one, we have for instance $\delta\left(e_{1}\right)=\left\{e_{1}, e_{2}, e_{3}, e_{4}\right\}, \delta\left(e_{2}\right)=\left\{e_{1}, e_{2}, e_{3}\right\}$, and for the second one, $\delta\left(e_{i}\right)=\left\{e_{1}, e_{2}, e_{2}\right\}$, for $i=1,2,3$.

Example 2. Another example, where less hyperedges are added, can be obtained by imposing a minimal cardinality on the intersection: $\forall e \in E, B_{e}^{k}=\left\{e^{\prime} \in\right.$ $\left.\mathcal{E}|| V(e) \cap V\left(e^{\prime}\right) \mid \geq k\right\}$.

Example 3. Let us now consider dilations from $\mathcal{T}=(\mathcal{P}(\mathcal{E}), \subseteq)$ into $\mathcal{T}^{\prime}=$ $(\mathcal{P}(\mathcal{V}), \subseteq)$. This will be useful later on when considering dual hypergraphs (see Section (5). Then the elementary dilation should map a hyperedge to a subset of 

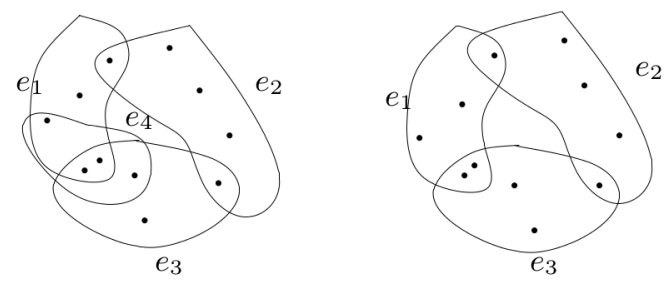

Fig. 1. Two hypergraphs $\left(V, E_{1}\right),\left(V, E_{2}\right)$, defined on the same set of vertices. Hyperedges are displayed as sets of vertices.

vertices. A trivial example is: $\forall e \in E, B_{e}=\delta(\{e\})=\{x \in \mathcal{V} \mid x \in e\}=V(e)$, where again $e$ is considered as a subset of vertices. This achieves the required mapping, but does not really dilate anything.

Example 4. More interesting, we can define a structuring element as in Example 1, but considering the resulting subset of vertices: $\forall e \in E, B_{e}=\delta(\{e\})=\{x \in$ $\mathcal{V} \mid \exists e^{\prime} \in \mathcal{E}, x \in e^{\prime}$ and $\left.V(e) \cap V\left(e^{\prime}\right) \neq \emptyset\right\}=\cup\left\{V\left(e^{\prime}\right) \mid V\left(e^{\prime}\right) \cap V(e) \neq \emptyset\right\}$. As in Example 2, we could add more strict constraints on the intersection, if we want the dilation to include less vertices.

Example 5. Let us now consider $\mathcal{T}=(\{H=(V, E)\}, \preceq)$. An elementary dilation can be defined according to the proposed canonical decomposition as: $\forall x \in V_{E}, \delta(\{x\}, \emptyset)=(\{x\}, \emptyset)$, for isolated vertices, and for elementary hypergraphs associated with hyperdeges: $\forall e \in E, \delta(V(e),\{e\})=\left(\cup\left\{V\left(e^{\prime}\right) \mid V\left(e^{\prime}\right) \cap\right.\right.$ $\left.V(e) \neq \emptyset\},\left\{e^{\prime} \in \mathcal{E} \mid V\left(e^{\prime}\right) \cap V(e) \neq \emptyset\right\}\right)$.

Note that if we consider also attributes on the vertices (or hyperedges), other examples can be provided by using a similarity between attributes. For instance isolated vertices could be dilated by adding all vertices that have similar attribute values.

\section{Dualities}

In the sequel $\delta(\{u\})$ will be simply denoted by $\delta(u)$.

Let $H=(V, E)$ be a hypergraph with $V \neq \emptyset, E \neq \emptyset$, and let $H^{*}=\left(V^{*}, E^{*}\right)$ its dual. Let also

$$
\delta: V \longrightarrow \mathcal{P}(V)
$$

be a mapping. From this mapping we define another one as:

$$
\begin{aligned}
& V \longrightarrow \mathcal{P}(V) \\
& x \longmapsto \delta^{*}(x)=\{y \in V ; x \in \delta(y)\}
\end{aligned}
$$

Let us notice that we can also define $\delta^{* *}$ as

$$
\begin{aligned}
& V \longrightarrow \mathcal{P}(V) \\
& x \longmapsto \delta^{* *}(x)=\left\{y \in V ; x \in \delta^{*}(y)\right\}
\end{aligned}
$$


The following proposition establishes basic results which will be useful next for deriving other results on duality, an important concept on hypergraphs. A particularly interesting result is the one expressed in Corollary 1 at the end of this section, linking morphological operators, derived rough spaces, and probability distributions.

Proposition 8. Let $H=(V, E)$ be a hypergraph with $V \neq \emptyset, E \neq \emptyset$ and $\delta$ and $\delta^{*}$ as introduced above; we have:

a) for all $X \in \mathcal{P}(V), \delta^{*}(X)=\bigcup_{x \in X} \delta^{*}(x)=\{y \in V, X \cap \delta(y) \neq \emptyset\}$ (resp. $\left.\delta(X)=\bigcup_{x \in X} \delta(x)=\left\{y \in V, X \cap \delta^{*}(y) \neq \emptyset\right\}\right)$ iff $\delta^{*}$ is a dilation (resp. $\delta$ is a dilation);

b) for all $X \in \mathcal{P}(V)$, if $\bigcup_{x \in X} \delta^{*}(x)=V$ (resp. $\bigcup_{x \in X} \delta(x)=V$ ) then $X \subseteq$ $\bigcup_{X \cap \delta^{*}(y) \neq \emptyset} \delta^{*}(y)$ (resp. $\left.X \subseteq \bigcup_{X \cap \delta(y) \neq \emptyset} \delta(y)\right)$;

c) $\delta^{* *}=\delta$ on $V$;

d) if $\delta^{* *}$ and $\delta$ are dilations then $\delta^{* *}=\delta$.

Proof. a) Assume that $\delta^{*}$ is a dilation. The first equality is obvious by definition. Let us show the second one. Let $y \in \delta^{*}(X)=\bigcup_{x \in X} \delta^{*}(x)$ then there is a $x \in X$ such that $y \in \delta^{*}(x) \Longleftrightarrow x \in \delta(y)$, so $y \in\{z \in V, X \cap \delta(z) \neq \emptyset\}$.

Let $X \in \mathcal{P}(V)$, and $y \in\{z \in V, X \cap \delta(z) \neq \emptyset\}$, there is $x \in X$ such that $x \in \delta(y) \Longleftrightarrow y \in \delta^{*}(x)$, consequently $y \in \bigcup_{x \in X} \delta^{*}(x)$.

Conversely, if the equalities hold, then it follows from the first one that $\delta^{*}$ commutes with the supremum, and is hence a dilation.

b) Obvious.

c) Let $z \in \delta^{* *}(x)$ then $x \in \delta^{*}(z)$, and therefore $z \in \delta(x)$.

In the same way $z \in \delta(x) \Longrightarrow x \in \delta^{*}(z)$ and $z \in \delta^{* *}(x)$. So $\delta^{* *}=\delta$ on $V$.

d) From the definition of a dilation.

Let $\delta: \mathcal{P}(V) \longrightarrow \mathcal{P}(V)$ be a dilation. It gives rise to a hypergraph $H_{\delta}=$ $\left(V,(\delta(x))_{x \in V}\right)$, where $\delta(x)$ is seen as a hyperedge built by the vertices defining $\delta(x)$. Now, let $H=(V, E)$ be a hypergraph. We can associate a dilation to $H$, for instance by considering the following structuring function:

$$
\begin{aligned}
& V \longrightarrow \mathcal{P}(E) \\
& x \longmapsto \delta(x)=\{e \in E ; x \in e\}
\end{aligned}
$$

Proposition 9. Let $\delta: V \longrightarrow \mathcal{P}(V)$ be a mapping and $H=(V, E)$ be an $h y$ pergraph $(V \neq \emptyset, E \neq \emptyset)$ without isolated vertex and without repeated hyperedge. We have: $H \simeq H_{\delta} \Longleftrightarrow H^{*} \simeq H_{\delta^{*}}$.

Proof. Suppose that $H \simeq H_{\delta}$. Because $H$ is without repeated hyperedge, if $x \neq y$ then $\delta(x) \neq \delta(y)$, i. e. $\delta(x)=\delta(y)$ implies that $x=y$, so $\delta$ is injective on $V$.

Let $H=(V ; E)$ and $H_{\delta}=\left(V_{\delta}=V ; E_{\delta}=(\delta(x))_{x \in V}\right)$ be hypergraphs. We have: $H \simeq H_{\delta} \Longleftrightarrow$ there a bijection $f: V \longrightarrow V_{\delta}$ such that $e \in E \Longleftrightarrow f(e)=\delta(x) \in$ $E_{\delta}, x \in V$. Since $H_{\delta}$ has no repeated hyperedge, notice that $\left.(\delta(x))_{x \in V}\right)$ is a set $\{\delta(x), x \in V\}$. 
It is known that $H \simeq H_{\delta} \Longleftrightarrow H^{*} \simeq H_{\delta}^{*}$, with $H^{*}=\left(V^{*} \simeq E ; E^{*} \simeq\right.$ $\left.\left(H(x)_{x \in V}\right)\right), H_{\delta}^{*}=\left(V_{\delta}^{*} \simeq(\delta(x))_{x \in V}\right) ; E_{\delta}^{*} \simeq\left(H(x)_{x \in V}\right)$. It is sufficient to show that $H_{\delta}^{*} \simeq H_{\delta^{*}}$, with $H_{\delta^{*}}=\left(V_{\delta^{*}} \simeq V ; E_{\delta^{*}} \simeq\left(\delta^{*}(x)\right) \in V\right)$. Let $g$ be a correspondence defined by:

$$
\begin{array}{ll}
\{\delta(y), y \in V\} & \longrightarrow V \\
\delta(x) & \longmapsto g(\delta(x))=x
\end{array}
$$

Since $\delta$ is injective, we have $\delta(x)=\delta(y) \Longrightarrow x=y=g(\delta(x))=g(\delta(y))$, this correspondence is well defined $i$. e. it is a mapping.

Clearly $g$ is surjective; moreover $g$ is injective since $|\{\delta(y), y \in V\}|=|V|$. Hence $g$ is a bijection.

Now, $H(x) \in E_{\delta}^{*} \Longleftrightarrow H(x)=\left\{\delta\left(u_{i}\right), x \in \delta\left(u_{i}\right)\right\}=\left\{\delta\left(u_{1}\right), \delta\left(u_{2}\right), \ldots \delta\left(u_{k}\right)\right\} \in$ $E_{\delta}^{*} \Longleftrightarrow g(H(x))=\left\{g\left(\delta\left(u_{i}\right)\right), i \in\{1,2,3, \ldots k\}\right\}=\left\{u_{1}, u_{2}, \ldots u_{k}\right\}=\delta^{*}(x)$, because $x \in \delta\left(u_{i}\right) \Longleftrightarrow u_{i} \in \delta^{*}(x)$.

Hence $H(x) \in E_{\delta}^{*} \Longleftrightarrow g(H(x))=\delta^{*}(x) \in E_{\delta^{*}}$. So $H_{\delta}^{*} \simeq H_{\delta^{*}}$, and finally $H \simeq H_{\delta} \Longleftrightarrow H^{*} \simeq H_{\delta^{*}}$.

Proposition 10. Let $H^{*}=\left(V^{*}, E^{*}\right)$ be a hypergraph and let $P=\left(p_{i}\right)_{i \in\{1,2, \ldots, t\}}$ be a discrete probability distribution on $V^{*}$, taking rational values. This probability distribution gives rise to a dilation, (resp. a erosion).

Let $\delta$ be a dilation on $V^{*}$, then this dilation gives rise to a discrete probability distribution on $V^{*}$.

Proof. To prove the proposition, we will exhibit a particular dilation from $P$, and respectively a particular probability distribution from a dilation.

Let $P=\left(p_{i}\right)_{i \in\{1,2, \ldots, t\}}$ be a discrete probability distribution with rationale values on $V^{*}$. For all $i \in\{1,2, \ldots, t\}$ there are $a_{i}, b_{i} \in \mathbb{N}, b_{i} \neq 0$, such that $p_{i}=\frac{a_{i}}{b_{i}}=$ $\frac{a_{i}\left|V^{*}\right|}{b_{i}\left|V^{*}\right|}=\frac{\frac{a_{i}\left|V^{*}\right|}{b_{i}}}{\left|V^{*}\right|}$. We have:

$1=\sum_{i} p_{i}=\sum_{i} \frac{\frac{a_{i}\left|V^{*}\right|}{b_{i}}}{\left|V^{*}\right|}=$

$\sum_{i}\left(\frac{\left\lfloor\frac{a_{i}}{b_{i}} \cdot\left|V^{*}\right|\right\rfloor}{\left|V^{*}\right|}\right)+\frac{\left|V^{*}\right|-\sum_{j}\left\lfloor\frac{a_{j}}{b_{j}} \cdot\left|V^{*}\right|\right\rfloor}{\left|V^{*}\right|}=\sum_{i}\left(\frac{\left\lfloor\frac{a_{i}}{b_{i}} \cdot\left|V^{*}\right|\right\rfloor+\left|V^{*}\right|-\sum_{j}\left\lfloor\frac{a_{j}}{b_{j}} \cdot\left|V^{*}\right|\right\rfloor}{\left|V^{*}\right|}\right)$.

Let $V_{1}^{*}=\left\{x_{1}^{*}, x_{2}^{*}, \ldots x_{\left\lfloor\frac{a_{1}}{b_{1}} \cdot\left|V^{*}\right|\right\rfloor}^{*}\right\}, V_{2}^{*}=\left\{x_{\left\lfloor\frac{a_{1}}{b_{1}} \cdot\left|V^{*}\right|\right\rfloor+1}^{*} \ldots x_{\left\lfloor\frac{a_{1}}{b_{1}} \cdot\left|V^{*}\right|\right\rfloor+\left\lfloor\frac{a_{2}}{b_{2}} \cdot\left|V^{*}\right|\right\rfloor}^{*}\right\} \ldots$ $V_{t+1}^{*}=V^{*} \backslash \bigcup_{i=1}^{t} V_{i}^{*}$. Without loss of generality, we can assume that $V_{i}^{*} \neq \emptyset$ for all $i \in\{1,2, \ldots, t+1\}$. By construction we have: $V_{i}^{*} \cap V_{j}^{*}=\emptyset$ for all $i, j \in$ $\{1,2, \ldots, t+1\}, i \neq j$. Consequently $\left(V_{i}^{*}\right)_{i \in\{1,2, \ldots, t+1\}}$ is a partition of $V^{*}$.

The hypergraph $H^{*}=\left(V^{*}, E^{*}\right)$ can be seen as a dual of a hypergraph $H=$ $(V, E)$. Because $V^{*} \simeq E \Longleftrightarrow \bigcup_{i=1}^{t+1} V_{i}^{*} \simeq \bigcup_{i=1}^{t+1} \bar{E}_{i}, K=\left(\bar{E}_{i}\right)_{i \in\{1,2, \ldots, t+1\}}$ is a partition of $E$, where $\bar{E}_{i}$ is a subset of $E$, dual of $V_{i}^{*}$. Let us define for $A \subseteq E$

$$
\varepsilon(A)=\left\{\bar{E}_{i} \in K ; \bar{E}_{i} \subseteq A\right\} \text { and } \delta(A)=\left\{\bar{E}_{i} \in K, \bar{E}_{i} \cap A \neq \emptyset\right\} .
$$

It is easy to verify that $\varepsilon$ is an erosion and $\delta$ a dilation from $(\mathcal{P}(E), \subseteq)$ into $(\mathcal{P}(\mathcal{P}(E)), \subseteq)$. 
Now, let $\delta$ be a dilation on $V^{*}$; the relation $x_{i}^{*} \sim_{\delta} y_{j}^{*} \Longleftrightarrow \delta\left(x_{i}^{*}\right)=\delta\left(y_{j}^{*}\right)$ is an equivalence relation on $V^{*}$. We then denote by $\bar{V}^{*}{ }_{i}$ the equivalence classes:

$$
V^{*} / \sim_{\delta}=\left\{{\overline{V^{*}}}_{i} ; i \in\{1,2, \ldots t\}\right\} .
$$

Let us now define $p_{i}=\frac{\left|\overline{V^{*}}\right|}{\left|\overline{V^{*}}\right|}$. We then have $0 \leq p_{i} \leq 1$ for all $i \in\{1,2, \ldots t\}$ and $\sum_{i} p_{i}=1$, thereby $\left(p_{i}\right)_{i \in\{1,2, \ldots t\}}$ is a discrete probability distribution.

This proposition is also interesting to establish links with rough sets. The definition of lower and upper approximations in terms of erosion and dilation, and the equivalence with rough sets have been developed in [23]. This result extends these notions to the case of hypergraphs, and $\varepsilon(A)$ and $\delta(A)$ exhibited in the proof are then lower and upper approximations of $A$ in a rough space (this is close to the approach proposed in [14]). Moreover, it adds a link with probabilities.

Corollary 1. Any discrete distribution of probability on $V^{*}$ gives rise to a rough space on $E$.

Conversely any rough space on $E$ gives rise to a discrete distribution of probability on $V^{*}$.

\section{Hypergraph Similarity Based on Dilations}

As an example of using mathematical morphology on hypergraphs, we briefly propose a notion of similarity between hypergraphs, based on dilations. It is well known that hypergraphs can be used to model several types of networks, such as biological, computer science, semantic networks 9,18,19. One of the most important tasks is to compare two networks. This comparison can be done using isomorphisms. However, there are two main drawbacks related to the use of isomorphisms:

- the first one concerns tractability, since there is no efficient algorithm to produce an isomorphism between two hypergraphs;

- the second one is that the isomorphism assumption is too rigid, and does not allow considering two hypergraphs as similar if they are not strictly isomorphic.

So we propose to define a new type of "comparator" between hypergraphs, based on dilation, which allows to introduce some "tolerance" for comparing sets of hyperedges, defining a similarity as a degree of overlap between dilated sets of hyperedges.

For any hypergraph $(V, E)$, we define a dilation on the hyperedges $E$, for example as:

$$
\begin{aligned}
& \mathcal{P}(E) \longrightarrow \mathcal{P}(E) \\
& A \quad \longmapsto \delta(A)=\{e \in E ; V(A) \cap e \neq \emptyset\}
\end{aligned}
$$

In the sequel we suppose that if $\delta(A)=\emptyset$ then $A=\emptyset$ (this typically holds when $\delta$ is extensive). 
Let $H^{1}=\left(V, E^{1}\right)$ and $H^{2}=\left(V, E^{2}\right)$ be two hypergraphs without empty hyperedge and $\delta_{E^{1}}$ and $\delta_{E^{2}}$ dilations defined on the set of hyperedges of $H^{1}$ and $H^{2}$, respectively. We define a similarity function $s$ by:

$$
\begin{aligned}
\mathcal{P}\left(E^{1}\right) \times \mathcal{P}\left(E^{2}\right) \backslash(\emptyset, \emptyset) & \longrightarrow \mathbb{R}^{+} \\
(A, B) & \longmapsto s(A, B)=\frac{\left|\delta_{E^{1}}(A) \cap \delta_{E^{2}}(B)\right|}{\left|\delta_{E^{1}}(A) \cup \delta_{E^{2}}(B)\right|}
\end{aligned}
$$

As an illustration, let us consider again the example in Figure1, with the definition of dilation as in Equation 8. We have quite high similarity values, which fit with the intuition, although the hypergaphs are not isomorphic: $s\left(e_{1}, e_{i}\right)=\frac{3}{4}$, $i \in\{1,2,3\} ; s\left(e_{2}, e_{i}\right)=\frac{3}{3}, i \in\{1,2,3\} ; s\left(e_{3}, e_{i}\right)=\frac{3}{4}, i \in\{1,2,3\} ; s\left(e_{4}, e_{i}\right)=\frac{2}{3}$, $i \in\{1,2,3\} ; s\left(\left\{e_{1}, e_{2}\right\} ; B\right)=s\left(\left\{e_{1}, e_{3}\right\} ; B\right)=s\left(\left\{e_{1}, e_{4}\right\} ; B\right)=s\left(\left\{e_{2}, e_{3}\right\} ; B\right)=$ $s\left(\left\{e_{2}, e_{4}\right\} ; B\right)=s\left(\left\{e_{3}, e_{4}\right\} ; B\right)=\frac{3}{4}$, for $B \subseteq E_{2}, B \neq \emptyset ; s\left(\left\{e_{1}, e_{2}, e_{3}\right\} ; B\right)=$ $s\left(\left\{e_{2}, e_{3}, e_{4}\right\} ; B\right)=s\left(\left\{e_{1}, e_{3}, e_{4}\right\} ; B\right)=s\left(\left\{e_{1}, e_{2}, e_{4}\right\} ; B\right)=\frac{3}{4}$, for $B \subseteq E_{2}, B \neq \emptyset$; and $s\left(\left\{e_{1}, e_{2}, e_{3}, e_{4}\right\} ; B\right)=\frac{3}{4}$, for $B \subseteq E_{2}, B \neq \emptyset$.

Proposition 11. Let $H^{1}=\left(V, E^{1}\right)$ and $H^{2}=\left(V, E^{2}\right)$ be two hypergraphs without empty hyperedge, and $\delta_{E^{1}}$ and $\delta_{E^{2}}$ extensive dilations (i.e. for each hyperegde $e$, we have $\left.e \in \delta_{E^{i}}(e)\right)$ defined on $E^{1}$ and $E^{2}$. We have the following properties:

a) $\forall\left(e_{i}, e_{j}\right) \in E^{1} \times E^{2}, s\left(\left(e_{i}, e_{j}\right)\right)=0 \Longleftrightarrow E^{1} \cap E^{2}=\emptyset$;

b) $\forall\left(e_{i}, e_{j}\right) \in E^{1} \times E^{2}, s\left(\left(e_{i}, e_{j}\right)\right)=1 \Longrightarrow E^{1}=E^{2}$,

c) $s$ is symmetrical.

Proof. a) $\forall\left(e_{i}, e_{j}\right) \in E^{1} \times E^{2}, s\left(\left(e_{i}, e_{j}\right)\right)=0 \Longleftrightarrow \forall\left(e_{i}, e_{j}\right) \in E^{1} \times E^{2}, \delta_{E^{1}}\left(e_{i}\right) \cap$ $\delta_{E^{2}}\left(e_{j}\right)=\emptyset \Longrightarrow \forall\left(e_{i}, e_{j}\right) \in E^{1} \times E^{2}, e_{i} \notin \delta_{E^{2}}\left(e_{j}\right)$ and $e_{j} \notin \delta_{E^{1}}\left(e_{i}\right)$ hence $E^{1} \cap E^{2}=\emptyset$. Indeed, since $\delta_{E^{2}}$ is extensive, $e_{j} \in \delta_{E^{2}}\left(e_{j}\right)$ and $\cup_{e_{j}} \delta_{E^{2}}\left(e_{j}\right)=E^{2}$, and therefore having $e_{i} \notin \delta_{E^{2}}\left(e_{j}\right)$ for all $e_{j}$ implies $e_{i} \notin E^{2}$. Similarly $e_{j} \notin E^{1}$. Conversely, if $E^{2} \cap E^{2} \neq \emptyset$, then $\mathcal{P}\left(E^{1}\right) \cap \mathcal{P}\left(E^{2}\right) \neq \emptyset$ and any $\delta_{E^{1}}(A)$ is disjoint from any $\delta_{E^{2}}(B)$.

b) $\forall\left(e_{i}, e_{j}\right) \in E^{1} \times E^{2}, s\left(\left(e_{i}, e_{j}\right)\right)=1 \Longleftrightarrow \forall\left(e_{i}, e_{j}\right) \in E^{1} \times E^{2} \delta_{E^{1}}\left(e_{i}\right)=\delta_{E^{2}}\left(e_{j}\right)$. Since $\delta$ is extensive, $\forall e_{i} \in E^{1}, e_{i} \in \delta_{E^{1}}\left(e_{i}\right)$, hence $e_{i} \in \delta_{E^{2}}\left(e_{i}\right)$ and therefore $e_{i} \in E^{2}$. Similarly $\forall e_{j} \in E^{2}, e_{j} \in E^{1}$. Therefore $E^{1}=E^{2}$.

c) The symmetry of $s$ is straightforward.

\section{Conclusion}

In this article we introduced mathematical morphology on hypergraphs. To show the relevance of this relationship between these two domains, we have exhibited a notion of duality in mathematical morphology which corresponds to the concept of duality which is important in the theory of hypergraphs. Other properties of hypergraphs can undoubtedly be expressed using morphological operators (probably such as transversal of a subset of hyperedges of a hypergraph, matching contained in a subset of vertices of a hypergraph). Future work will aim on the one hand at exploring such properties, and on the other hand at studying in more depth the concept of similarity. 


\section{References}

1. Berge, C.: Hypergraphs. Elsevier Science Publisher, The Netherlands (1989)

2. Bloch, I.: On Links between Mathematical Morphology and Rough Sets. Pattern Recognition 33(9), 1487-1496 (2000)

3. Bloch, I., Heijmans, H., Ronse, C.: Mathematical Morphology. In: Aiello, M., PrattHartman, I., van Benthem, J. (eds.) Handbook of Spatial Logics, ch. 13, pp. $857-$ 947. Springer, Heidelberg (2007)

4. Bretto, A., Azema, J., Cherifi, H., Laget, B.: Combinatorics and image processing. CVGIP: Graphical Model and Image Processing 59(5), 265-277 (1997)

5. Bretto, A., Cherifi, H., Aboutajdine, D.: Hypergraph imaging: an overview. Pattern Recognition 35(3), 651-658 (2002)

6. Cousty, J., Najman, L., Serra, J.: Some morphological operators in graph spaces. In: Wilkinson, M.H.F., Roerdink, J.B.T.M. (eds.) ISMM 2009. LNCS, vol. 5720, pp. 149-160. Springer, Heidelberg (2009)

7. Heijmans, H.J.A.M.: Morphological Image Operators. Academic Press, Boston (1994)

8. Heijmans, H.J.A.M., Ronse, C.: The Algebraic Basis of Mathematical Morphology - Part I: Dilations and Erosions. Computer Vision, Graphics and Image Processing 50, 245-295 (1990)

9. Klamt, S., Haus, U.U., Theis, F.: Hypergraphs and cellular networks. PLoS Comput. Biol. 5(5) (2009)

10. Meyer, F., Stawiaski, J.: Morphology on graphs and minimum spanning trees. In: Wilkinson, M.H.F., Roerdink, J.B.T.M. (eds.) ISMM 2009. LNCS, vol. 5720, pp. 161-170. Springer, Heidelberg (2009)

11. Ronse, C., Heijmans, H.J.A.M.: The Algebraic Basis of Mathematical Morphology - Part II: Openings and Closings. Computer Vision, Graphics and Image Processing 54, 74-97 (1991)

12. Serra, J.: Image Analysis and Mathematical Morphology. Academic Press, New-York (1982)

13. Serra, J. (ed.): Image Analysis and Mathematical Morphology, Part II: Theoretical Advances. Academic Press, London (1988)

14. Stell, J.: Relational Granularity for Hypergraphs. In: Szczuka, M., Kryszkiewicz, M., Ramanna, S., Jensen, R., Hu, Q. (eds.) RSCTC 2010. LNCS (LNAI), vol. 6086, pp. 267-276. Springer, Heidelberg (2010)

15. Ta, V.-T., Elmoataz, A., Lézoray, O.: Partial difference equations over graphs: Morphological processing of arbitrary discrete data. In: Forsyth, D., Torr, P., Zisserman, A. (eds.) ECCV 2008, Part III. LNCS, vol. 5304, pp. 668-680. Springer, Heidelberg (2008)

16. Vincent, L.: Graphs and Mathematical Morphology. Signal Processing 16, 365-388 (1989)

17. Voloshin, V.I.: Introduction to Graph and Hypergraph Theory. Nova Science, Bombay (2009)

18. Yang, M., Yang, Y.: A hypergraph approach to linear network coding in multicast networks. IEEE Transactions on Parallel and Distributed Systems 21, 968-982 (2010)

19. Zhen, L., Jiang, Z.: Hy-SN: Hyper-graph based semantic network. KnowledgeBased Systems 23(8), 809-816 (2010) 